\title{
Skin sensitivity - extremely positive reaction for nickelsulfate hexahydrate in association with occupational allergens
}

\author{
Donka A. Brambarova1, Rumyana K. Yankova ${ }^{1,2}$ \\ ${ }^{1}$ Department of Dermatology and Venereology, Faculty of Medecine, Medical University, Plovdiv, Bulgaria, ${ }^{2}$ Head of \\ Dermovenereology and Allergology Center, UMBAL'Pulmed', Plovdiv, Bulgaria
}

Corresponding author: Dr. Donka A. Brambarova, E-mail: d.brambarova@abv.bg

Sir,

Cosmetic products are frequent causative agents of cutaneous reactions due to changed sensitivity. We report an unexpected extremely positive reaction of Patch test for Nickelsulfate hexahydrate in association with others positive occupational allergens that needs of immediate treatment and interrupted the further exanimations.

The subject is 35 years old woman, worked like hairdresser from 6 years. She has history for itchy redness legions with desquamation on the skin of the face where applying different cosmetic products and on the neck after caring jewels of base metals from few years. She has itchy redness legions on the hands from four months with temporal effect of therapy with topical corticosteroids. No history of urticaria and atopy. The subject is healthy at the moment of examination, without any skin lesions and meet the following requirements for testing: good general condition, without serious common diseases and contraindications to conduct testing (acute myocardial infarction, decompensated diabetes, decompensated thyrotoxicosis, renal failure, active tuberculosis, pregnancy), without skin rash (acute and chronic eczema, urticaria, fungal infection, etc.) who do not accept and topically applied drugs affecting the result of testing (corticosteroids, antihistamines, tranquillizers, sympathomimetics). We perform Patch test with European Baseline Series and Hairdressing Series (Chemotechnique Diagnostics, Sweden) to diagnose contact sensitivity to locally administered funds.
European Baseline Series is a basic 'standard' series consist of allergens based on the experience of the European Environmental and Contact Dermatitis Research Group (EECDRG). Hairdressing Series contains different chemicals (metals, dyes, stabilizers, preservatives) which one might risk getting exposed to working professionally in the hairdressing occupation. Standardization is the result of years of testing large numbers of individuals, and it reflects a balance of sensitivity and specificity (ie, positive test results in the vast majority of allergic individuals and few positive findings in controls) [1].

Allergens in standard concentrations are placed in standard hypoallergenic chambers IQ Ultra (Chemotechnique Diagnostics, Sweden) with defined test areas (critical for standardization as the concept of dose/area) on the skin of the back and were removed after 48 hours.

The basic requirements for a patch-test chamber are an inert material applied to a hypoallergenic tape for providing good occlusion and fixation of the test unit to the skin [1].

The results are reported at 48 hour and at 72 hour. The reactions are reported as required by the International Contact Dermatitis Research Group (ICDRG).

\section{Results of Patch Test}

Positive reactions on 48 hour are four and one is doubtful: Nickelsulfate hexahydrate $(+++)$,

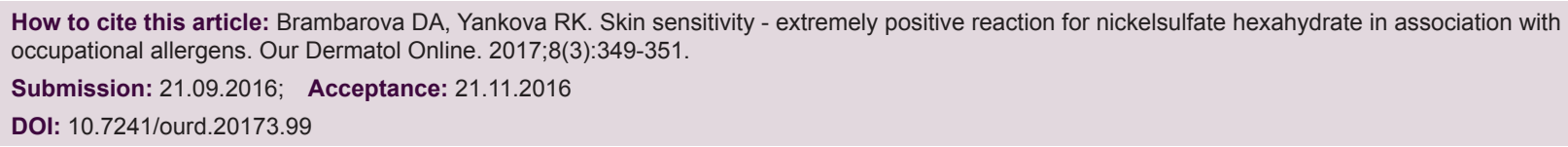


2-Mercaptobenzothiazole $(++)$, Captan $(+)$, Diazolidinyl urea $(+)$, p-Phenylenediamine base $($ ? + ). For Nickelsulfate hexahydrate on 48 hour positive result is extremely positive and the subject has itching and very high disturbance on the place of the probe (Fig. 1).

The doubtful reaction $(?+)$ for p-Phenylenediamine base (PPD) is positive on 72 hour. So the positive reactions on 72 hour are five: Nickelsulfate hexahydrate $(+++)$, 2-Mercaptobenzothiazole $(++)$, Captan $(+)$, Diazolidinyl urea $(+)$ and PPD $(+)$. For Nickelsulfate hexahydrate positive result on 72 hour is with crescendo phenomenon: increasing erythema, infiltration and exudation, increased in size, merged in nearly horizontal line with the opposite allergen, very disturbing itch. Crescendo phenomenon is specific for allergic reactions and is the main deferens of irritant reactions.

Because of the extremely positive and disturbing reaction for Nickelsulfate hexahydrate next readings on 96 hours and on 7 day are not performed. An immediate treatment is started with topical corticosteroids twice daily and antihistamines p.o. ones daily for a week.

\section{CONCLUSION AND DISCUSSION}

We report extremely positive reaction with crescendo phenomenon for Nickelsulfate hexahydrate in association with others positive allergens in European Baseline Series and positive occupational allergens in Hairdressing Series. Nickelsulfate hexahydrate is a very frequent allergen $[2,3]$. In the last 5 years he is the most frequent allergen in our practice but extremely positive reactions we met are very rare. In our case the positive reaction is so extremely that the subject needs

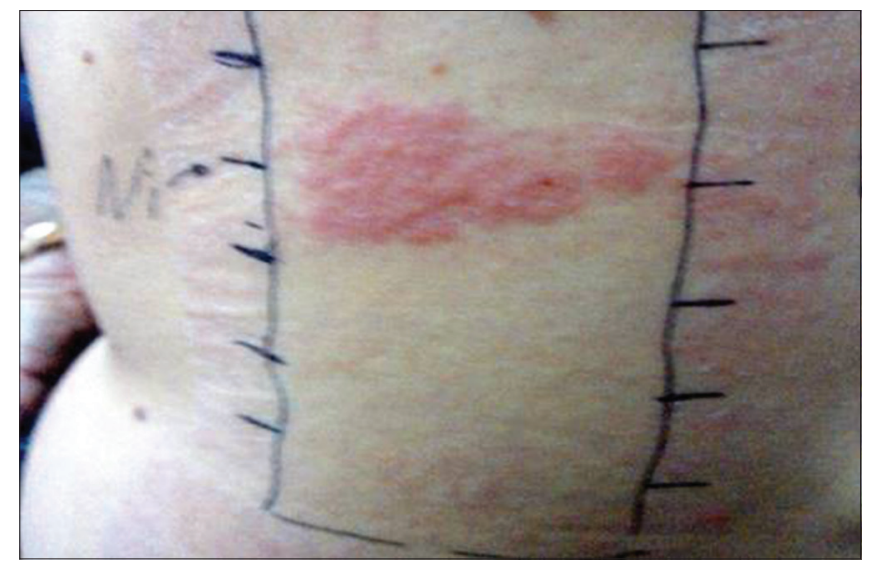

Figure 1: Positive reactions on 48 hour: Nickelsulfate hexahydrate $(+++)$ and 2-Mercaptobenzothiazole (++). of immediate treatment and readings on 96 hours and on 7 day are not performed. Late patch-test readings are useful when interpreting reactions to metals [1].

Nickelsulfate hexahydrate and PPD are more frequent allergens in woman what is in our case. Nickelsulfate hexahydrate remains the most common contact sensitizer and is found more frequently in women than in men. The gender difference is likely due to greater exposure of the neck, hands, and ears to nickel in jewelry [4,5]. PPD is common allergen in cosmetics associated with hair care products [6]. PPD is the active ingredient in many permanent and semi-permanent hair dyes. It is a major cause of Allergic Contact Dermatitis (ACD) among hairdressers. There are another two positive reactions in Hairdressing Series, because of professional exposure - Captan and Diazolidinyl urea.

Occupational contact dermatitis (OCD) is a contact dermatitis in a patient who presents with dermatitis associated with workplace exposures [4]. In addition to the most common hair dye sensitizer (PPD), there are sensitizers in shampoos, including fragrances, $\mathrm{CAPB}$, and preservatives. There are seven criteria in our case of Mathias's seven criteria for confirmation of OCD: 1. the clinical appearance that is consistent with CD; 2. potential culprit cutaneous irritants and/or allergens are present in the workplace; 3 . the anatomic distribution of dermatitis is consistent with workplace skin exposure; 4. the temporal relationship between exposure and onset of symptoms is consistent with CD; 5. nonoccupational exposures are excluded as probable causes of the dermatitis; 6 . the dermatitis improves when absent from work exposure, and re-exposure results in exacerbation; 7 . Patch test performed according to established guidelines demonstrates positive and relevant reactions.

Diagnostic of OCD is difficult. Patch test as a classical method for the diagnostic of contact allergy is an easy and a safety method. Side effects of Patch test are unusual but strong positive reactions could be disturbing and harmful for the patients. In our case the extremely positive result for Nickelsulfate hexahydrate on 72 hour with crescendo phenomenon needs of a treatment and interrupt the further readings.

\section{REFERENCES}

1. Wolf R, Orion E, Ruocco V, Baroni A, Ruocco E. Patch testing: Facts and controversies. Clin Dermatol. 2013;31:479-86.

2. Aguilar-Bernier M, Bernal-Ruiz AI, Rivas-Ruiz F, Fernández- 
www.odermatol.com

Morano MT, de Troya-Martín M. Contact Sensitization to Allergens in the Spanish Standard Series at Hospital Costa del Sol in Marbella, Spain: A Retrospective Study (2005-2010). Actas Dermosifiliogr. 2012;103:223-8.

3. Balato A, Balato N, Costanzo L, Ayala F. Contact sensitization in the elderly. Clin Dermatol. 2011;29:24-30.

4. Fonacier L, Bernstein DI, Pacheco K, Holness DL, Blessing-Moore J, Khan D, et al. Contact dermatitis: a practice parameter - update 2015. J Allergy Clin Immunol Pract. 2015;3:S1-39.

5. Ruff $\mathrm{C}$, Belsito $\mathrm{D}$. The impact of various patient factors on contact allergy to nickel, cobalt, and chromate. J Am Acad Dermatol. 2006;55:32-9.
6. Warshaw EM, Buchholz HJ, Belsito DV, Maibach HI, Fowler JF Jr, Rietschel RL, et al Allergic patch test reactions associated with cosmetics: Retrospective analysis of cross-sectional data from the North American Contact Dermatitis Group, 2001-2004. J Am Acad Dermatol. 2009;60:23-38.

Copyright by Donka A. Brambarova, et al. This is an open-access article distributed under the terms of the Creative Commons Attribution License, which permits unrestricted use, distribution, and reproduction in any medium, provided the original author and source are credited.

Source of Support: Nil, Conflict of Interest: None declared. 